\title{
(-)Epigallocatechin-3-Gallate Directly Inhibits MT1-MMP Activity, Leading to Accumulation of Nonactivated MMP-2 at the Cell Surface
}

\author{
Isabella Dell'Aica, Massimo Donà, Luigi Sartor, Elga Pezzato, and \\ Spiridione Garbisa
}

Department of Experimental Biomedical Sciences, Medical School of Padova, Padova, Italy

\begin{abstract}
SUMMARY: Consumption of green tea has been associated with prevention of cancer development, metastasis, and angiogenesis. Given the crucial role of the matrix metallo-proteinase-2 (MMP-2) on the degradation of the extracellular matrix instrumental to invasion, we examined the effect of the main flavanol present, (-)epigallocatechin-3-gallate (EGCG), on membrane-type 1 MMP (MT1-MMP), the receptor/activator of MMP-2. In-solution fluorimetric assay with activated MT1-MMP and gelatin-zymography with MT1-MMP catalytic domain alone and pro-MMP-2 activation by the same domain revealed dose-dependent inhibition of MT1-MMP at EGCG concentrations slightly lower than that reported to inhibit MMP-2 and MMP-9. Cytofluorimetry and immunolocalization revealed that EGCG does not impair MT1-MMP/TIMP-2/MMP-2 presence on the cell membrane. In the membrane extract of HT-1080 human fibrosarcoma cells, $10 \mu \mathrm{M}$ EGCG caused a strong increase in MT1-MMP level and accumulation of pro-MMP-2 while leaving activated MMP-2 unchanged. EGCG thus exerts inhibition of MT1-MMP, which restrains activation of MMP-2; this may confer the antiangiogenic and antimetastatic activity associated with green tea. (Lab Invest 2002, 82:1685-1693).
\end{abstract}

A number of epidemiological observations have led to the association of green tea consumption with health benefits, including decreased risk of cardiovascular diseases and cancer development and metastasis (Yang and Wang, 1993). Experimental studies showing the beneficial properties of green tea and its components are increasingly reported in the scientific literature; these include inhibition of mutagenesis (Wang et al, 1989), tumorigenesis (Yang and Wang, 1993), tumor growth (Wang et al, 1992), invasion (Garbisa et al, 2001; Sazuka et al, 1995), metastasis (Taniguchi et al, 1992), and angiogenesis (Cao and Cao, 1999).

These effects have largely been attributed to the most prevalent polyphenol contained in green tea, the catechin (-)epigallocatechin-3-gallate (EGCG). EGCG has been shown to directly inhibit matrix metalloproteinase-2 (MMP-2) and MMP-9 gelatinases (Demeule et al, 2000; Garbisa et al, 1999; MaedaYamamoto et al, 1999), the two matrix metalloproteinases that are most frequently overexpressed in cancer and angiogenesis and that are instrumental in cutting through basement membrane barriers (Liotta

DOI: 10.1097/01.LAB.0000043122.00384.91

Received September 9, 2002.

This research was supported by grants from Associazione Italiana per la Ricerca sul Cancro, MIUR Italian Government, and the University of Padova, Padova, Italy.

Address reprint requests to: Prof. S. Garbisa, Department of Experimental Biomedical Sciences, viale G. Colombo 3, 35121 Padova, Italy. E-mail: garbisa@unipd.it et al, 1980). This inhibition is paralleled by inhibition of reconstituted basement membrane invasion (Garbisa et al, 1999), which occurs in vitro at concentrations equivalent to those in the plasma of moderate green tea drinkers (Yang and Wang, 1993).

Although the molecular mechanisms responsible for the direct inhibition of the gelatinases instrumental to cancer invasion and angiogenesis are still to be elucidated, a number of enzymatic activities have been recently added to the list of those sensitive to catechin inhibition. These include the metallo-elastase secreted by macrophages (MMP-12) (Demeule et al, 2000), thrombin (Deana et al, unpublished data; Sartor et al, 2000b), and neutrophil serine-elastase (Sartor et al, 2002b). The latter, which is inhibited the most by EGCG $\left(\mathrm{IC}_{50} 0.3 \mu \mathrm{M}\right)$, may be instrumental in the invasion process because it can both directly contribute to basement membrane collagen degradation and also act as pro-MMP activator (Sternlicht and Werb, 1999).

Activation of gelatinases is in fact one of the crucial steps of the enzymatic cascade leading to extracellular matrix degradation and can be achieved by different proteases that cut off the N-terminal pro-peptide (Mazzieri et al, 1997; Sato et al, 1994). One of these is the membrane-type 1 matrix metallo-proteinase MT1MMP (Sato et al, 1994), which possesses a plasma membrane-spanning domain and short cytoplasmic tail at the C-terminal end. It can act as a receptor for tissue inhibitor of metallo-proteinases TIMP-2 or TIMP-3, which in turn bind secreted pro-MMP-2 and pro-MMP-13; the pro-MMPs are then activated at the cell surface, perhaps by the same complexed MT1- 
MMP or else monomeric MT1-MMP lacking bound inhibitors (Sternlicht and Werb, 1999). The receptoractivator MT1-MMP has been implicated as a key player in a proteinase cascade involving MMPs 2, 9, and 13 (Caenazzo et al, 1998; Cowell et al, 1998), which are thus acting at the cell-surface level by a cell-controlled mechanism functional to matrix degradation and invasion.

We have already reported that EGCG up-regulates the expression of MT1-MMP by human fibrosarcoma cells without enhancing gelatinase activation but with restraint of in vitro invasion (Garbisa et al, 2001), and other authors have shown that activation of MMP-2 is blocked by EGCG (Demeule et al, 2000). Recently, inhibition of MT1-MMP-driven migration has been described in glioblastoma cells (Annabi et al, 2002). Nevertheless, no evidence has yet been produced on direct impairment of this pivotal membrane proteinase by the catechin. In order to verify whether also MT1MMP receptorial-enzymatic activities are directly affected by EGCG, we investigated the effects of this phytofactor on the complete proteinase and the portion containing the active site as well as on MT1-MMP/ TIMP-2/MMP-2 colocalization.

\section{Results}

\section{Effect of EGCG on MT1-MMP Activity}

To study the possible inhibitory effects of EGCG on MT1-MMP, the activity of both the complete $p$-APMAactivated pro-MT1-MMP and the catalytic domain were studied in the presence of increasing concentrations of the flavanol.

Gelatin-zymography revealed that incubation of p-APMA-activated pro-MT1-MMP with pro-MMP-2containing serum-free culture medium failed to activate this zymogen (not shown). On the contrary, when a synthetic coumarin-labeled substrate was used, clear degradation was achieved and a dose-response inhibition by EGCG revealed: MT1-MMP activity was reduced by over $50 \%$ with $10 \mu \mathrm{M}$ and almost completely abolished with $100 \mu \mathrm{M}$ catechin (Fig. 1). The inhibition was maintained with a constant slope over the 30-minute period of the assay.

When gelatin was used as a natural substrate, the soluble p-APMA-activated pro-MT1-MMP failed to show any gelatinolytic activity (not shown), while the catalytic domain of MT1-MMP (MT1-MMP ${ }_{\text {cat }}$ ) produced a clear band of degradation (Fig. 2, control); in the latter case, 0.1 to $1000 \mu \mathrm{M}$ concentration of EGCG exerted dose-dependent inhibition of MT1-MMP activity, with an $\mathrm{IC}_{50}$ of approximately $5 \mu \mathrm{M}$ (Fig. 2).

The potential inhibition of MT1-MMP activity by EGCG was further studied by first incubating MT1$\mathrm{MMP}_{\text {cat }}$ with pro-MMP-2-containing medium-pretreated with proteinase inhibitors PMSF and NEMand verifying by gelatin-zymography the conversion of the 72-kd pro-MMP-2 to the 64-kd activated form. After 2 hours of incubation, the 72-kd form was better preserved in the presence of inhibitors than in their absence, and the conversion to the activated form

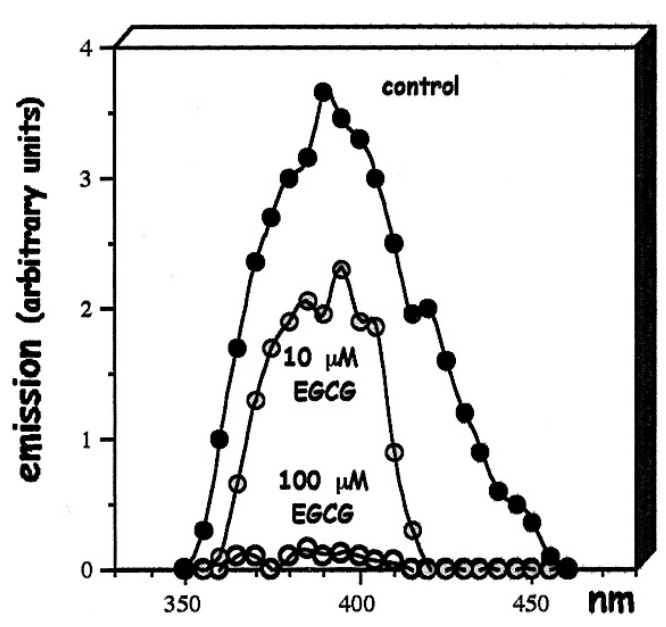

Figure 1.

Dose-dependent inhibition of MT1-MMP ( $p$-APMA-activated pro-enzyme) activity on synthetic substrate by EGCG. The fluorescent emission of the digested substrate falls with increasing concentrations of catechin. Example of duplicate experiment after background subtraction.
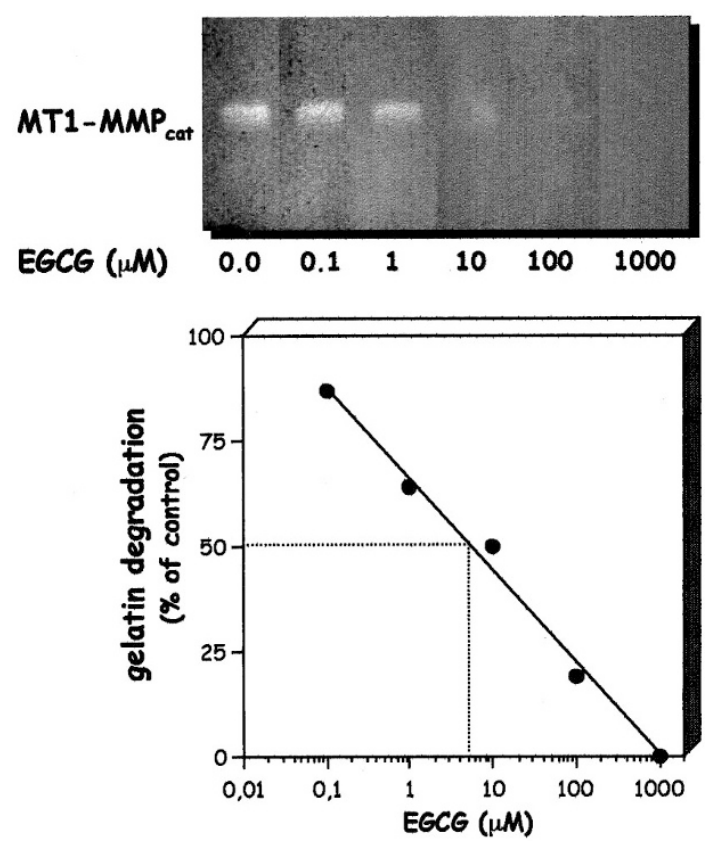

Figure 2

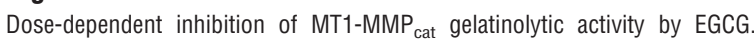
Gelatin zymography developed in the presence of increasing concentrations of catechin shows a progressively faint digestion band, with an $\mathrm{IC}_{50}$ of approximately $5 \mu \mathrm{m}$. Example of triplicate experiment.

was clearly obtained using 8 to $40 \mu \mathrm{U}$ of MT1-MMP cat $_{\text {ch }}$ (Fig. 3). After 4 hours of incubation, the 64-kd form did not undergo further modification (not shown).

When a $120 \mu \mathrm{U}$ dose was preincubated 20 minutes with 20 and $60 \mu \mathrm{M}$ EGCG and then incubated 4 hours with pro-MMP-2, triplicate experiments showed that conversion to the activated form of MMP-2 was almost completely inhibited even by the lowest catechin concentration (Fig. 4). The pro-MMP-2 gelatinolytic activity was well preserved in the MT1-MMP ${ }_{\text {cat }} /$ EGCG-containing samples, in comparison with the 


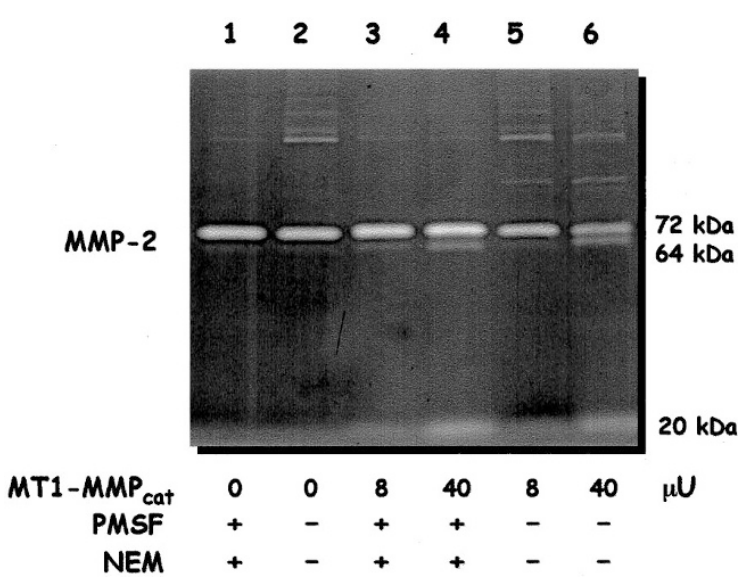

Figure 3.

MT1-MMP ${ }_{\text {cat }}$-induced activation of pro-MMP-2 (gelatin zymography). Medium conditioned by SK-N-BE neuroblastoma cells was used as the source of pro-MMP-2. Medium alone (control) incubated (1) with and (2) without PMSF and NEM; as in lane 1, in the presence of $8 \mu \mathrm{U}(3)$ and $40 \mu \mathrm{U}$ (4) MT1-MMP cat; as in lane 2, in the presence of $8 \mu \mathrm{U}$ (5) and $40 \mu \mathrm{U}(6) \mathrm{MT}^{-}-\mathrm{MMP}_{\text {cat. }}$. When MT1-MMP cat is added, the MMP-2 zymogen $(72 \mathrm{kd})$ is processed to the activated form $(64 \mathrm{kd})$ in a dose-dependent manner. Example of duplicate experiment.

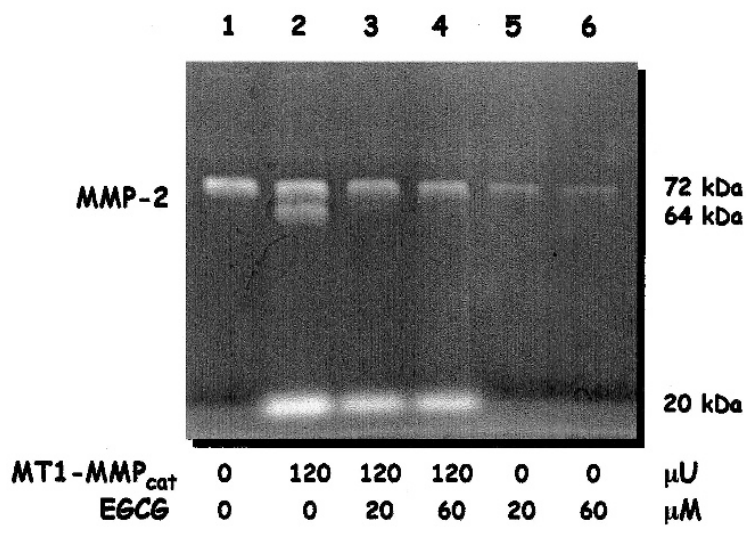

Figure 4.

MT1-MMP ${ }_{\text {cat }}$ preincubated with EGCG fails to activate pro-MMP-2 (gelatin zymography). (1) Pro-MMP-2 control; (2) pro-MMP-2 plus MT1-MMP cat $_{\text {; }}$ pro-MMP-2 plus MT1-MMP cat preincubated with $20 \mu \mathrm{M}$ (3) and $60 \mu \mathrm{M}$ (4) EGCG; pro-MMP-2 incubated with $20 \mu \mathrm{M}$ (5) and $60 \mu \mathrm{M}$ (6) EGCG. The gelatin zymography shows that pro-MMP-2 activation (generation of the 64-kd

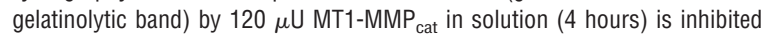
when the latter has been preincubated 20 minutes with 20 and $60 \mu \mathrm{M}$ EGCG (lanes 3 and 4). Example of triplicate experiment.

control containing EGCG but not MT1-MMP ${ }_{\text {cat }}$, where the activity was severely restrained (22 and $10 \%$ of control for the two concentrations). Also, the MT1$\mathrm{MMP}_{\text {cat }}$ band $(20 \mathrm{kd})$, visible at the bottom of the gel (Fig. 4), showed partially reduced gelatinolytic activity (69 and $80 \%$ of control) when the protease was incubated in presence of EGCG.

\section{Effect of EGCG on Cellular MMP Activities}

MMP-2-expressing cells (HT-1080) were also exposed to EGCG for 24 hours in gelatinase-free hi-FCSsupplemented medium: The cell membrane extract was analyzed by Western blotting for expression of
MT1-MMP and modulation of the MMP-2 zymogen/ activated form ratio.

As shown in the example in Figure $5 \mathrm{~A}$, the control human recombinant MT1-MMP was recognized by monoclonal $\mathrm{Ab}$ as a double band of 66 and $60 \mathrm{kd}$, corresponding $\mathrm{M}_{\mathrm{r}}$. In comparison with the control sample (without EGCG), the membrane extract of cells incubated with $10 \mu \mathrm{M}$, but not $20 \mu \mathrm{M}$, flavanol showed a strong positive band of $60 \mathrm{kd}$. A band with lower $\mathrm{M}_{\mathrm{r}}$ remains to be identified in the membrane extract, but its expression was not modulated by the phytofactor.

While with $10 \mu \mathrm{M}$ EGCG, an increased presence of pro-MMP-2 at the membrane level was also registered-which paralleled the up-regulation of MT1MMP-the amount of the MMP-2-activated form remained unmodified (Fig. 5B).

\section{Effect of EGCG on MT1-MMP/TIMP-2/MMP-2 Trimer}

To determine whether EGCG affects the MT1-MMP/ TIMP-2/MMP-2 complex, we first studied by cytofluorimetry the presence of these antigens on HT-1080 invasive cells (Mazzieri et al, 1997) treated with different concentrations of EGCG. When the cells were first exposed to the catechin, then rinsed and treated with the first antibody, no variations were registered on the positivity for either TIMP-2 and MMP-2 compared with the control (Fig. 6, A and C). When the sequence of treatments was reversed, a moderate but significant ( $p$ $=0.001$ ) decrease was observed in the number of cells positive for either TIMP-2 or MMP-2 (Fig. 6, B and $D$ ). Isotype controls were negative (not shown).

The potential displacement of TIMP-2 and/or MMP-2 from the cell membrane was further verified by immunocytological analysis on larger invasive cells, TRAMP-C2 (Ghosh et al, 1999). Confocal immunomicroscopy showed no detectable effect of EGCG (10 $\mu \mathrm{M})$ on the localization of the three antigens-MT1MMP, TIMP-2, MMP-2-compared with control (Fig. 7). They remained colocalized, mostly concentrated in just a few protruding cellular cones or peripheral caveolae, sometimes spottily distributed on the entire membrane, as seen in the dividing cell in Figure 7.

\section{Discussion}

The lack of increased activation of pro-MMP-2 in the presence of overexpression of the membrane-type 1 matrix metallo-proteinase (MT1-MMP), which acts as activator of MMP-2, and the fact that MT1-MMP belongs to a class of proteinases of which members are inhibited by EGCG (Demeule et al, 2000; Garbisa et al, 1999; Maeda-Yamamoto et al, 1999), led us to hypothesize a potential direct interaction of EGCG with MT1-MMP (Garbisa et al, 2001). More recently, this hypothesis was substantiated by indirect evidence that EGCG inhibits (a) pro-MMP-2 activation, (b) MT1-MMP processing to inactive form, and (c) MT1-MMP-driven migration in glioblastoma cells (Annabi et al, 2002). In this study, we demonstrate that micromolar concentrations of EGCG directly inhibit in a dose-dependent manner the enzymatic activity of 


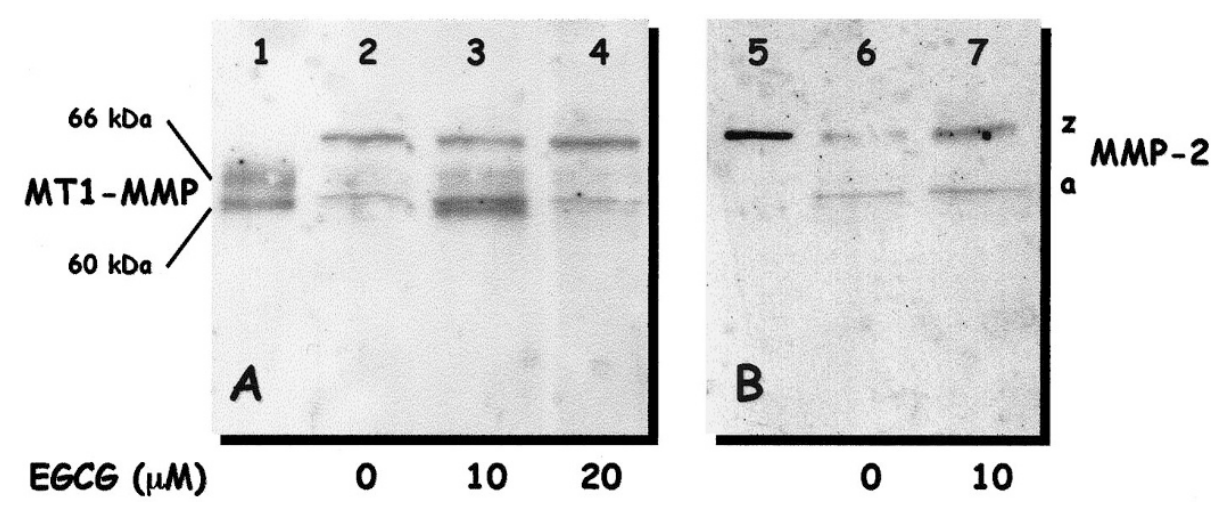

Figure 5.

Modulation of MT1-MMP (A) and MMP-2 (B) protein levels on cell membranes of HT-1080 incubated 24 hours in the presence of EGCG (Western blotting following 10 and $8 \%$ SDS-PAGE in A and B, respectively). (A) Human recombinant MT1-MMP control (1); membrane extract of cells cultured in the absence (2) or presence of 10 (3) and $20 \mu \mathrm{M}$ (4) EGCG. (B) Culture medium containing pro-MMP-2 as a control (5); membrane extract of HT-1080 cells incubated 24 hours in the absence (6) or presence of $10 \mu \mathrm{M}$ EGCG (7) containing both the zymogen (z) and the activated (a) form of MMP-2. Examples of duplicate experiment where loading has been normalized for protein content.

MT1-MMP without impairing the capacity of binding its ligand TIMP-2 and the third component of the membrane matrix-degradative complex, MMP-2.

The recombinant activated form of MT-1-MMP was ineffective in either activating in solution the zymogen form of MMP-2 or degrading gelatin in zymography, probably because of its transmembrane-protein nature to which correct stereo structure and function are linked. On the contrary, it has been reported that secreted MT1-MMP and an MT1-MMP deletion mutant lacking the transmembrane domain can cleave a number of extracellular substrates, including proMMP-2 and gelatin (Pei and Weiss, 1996). Four different assays developed in the presence of increasing EGCG concentrations were then used in our approach: (a) use of soluble $p$-APMA-activated MT1MMP on a synthetic fluorogenic substrate for matrix metallo-proteinases (Knight et al, 1992), (b) use of the MT1-MMP catalytic domain alone (MT1-MMP cat $)$ on gelatin, (c) use of MT1-MMP ${ }_{\text {cat }}$ on pro-MMP-2 in solution, (d) Western blot analysis of MT1-MMP and MMP-2 on cell lysates. Directly ( $a$ and $b$ ) or indirectly (c and d), all four assays revealed inhibition of MT1MMP activity by EGCG.

The degree of inhibition measured in biochemical assays $\left(\mathrm{IC}_{50}=5 \mu \mathrm{M}\right)$ is slightly higher than those recently reported for other metallo-proteinases (Demeule et al, 2000; Garbisa et al, 1999)-the gelatinases MMP-2 $(10 \mu \mathrm{M})$ and MMP-9 $(30 \mu \mathrm{M})$-but lower than those for the macrophage metallo-elastase MMP-12 (1 $\mu \mathrm{M})$ (Demeule et al, 2000) and the leukocyte serine-elastase $(0.3 \mu \mathrm{M})$ (Sartor et al, 2002b). Thus, the inhibition exerted by the flavanol on this pivotal receptor-activator, MT1-MMP, takes place at EGCG concentrations close to those physiologically reachable in the blood of moderate green tea drinkers (Yang et al, 2001).

In solution, MT1-MMP ${ }_{\text {cat }}$ produced the conversion of the MMP-2 zymogen into its activated form. The activation was almost completely inhibited even at 20 $\mu M$ EGCG (Fig. 4). This result was obtained with experiments carried out under conditions designed to maximize the preservation of enzymatic activities, ie, in the presence of serine- and thiol-proteinase inhibitors and at a lower temperature $\left(33^{\circ} \mathrm{C}\right)$ than that used in a recent report (Toth et al, 2002) but using a 60 -fold concentration of MT1-MMP cat for a shorter incubation period (4 versus 22 hours). With these precautions, the presence/activity of the 72- and 64-kd forms of MMP-2 was well preserved and the activity of the truncated form of MT1-MMP clearly resulted in the cleavage of the MMP-2 pro-peptide, but this proteoIytic activation was inhibited by EGCG. Although inhibition of other MT1-MMP-activated MMP-2-activating metalloproteinases present in the conditioned medium cannot be excluded, the demonstrated direct inhibition of MT1-MMP must also play a role in this case.

Control samples containing pro-MMP-2 and EGCG revealed that the moderate decrease of the gelatinoIytic activity in MT1-MMP cat $^{-}$-containing samples is not comparable with the strong decrease registered in controls without MT1-MMP cat (Fig. 4). This suggests that, after preincubation with MT1-MMP ${ }_{\text {cat }}$, less EGCG is available for binding to pro-MMP-2; the lack of the MMP-2-activated form in the presence of MT1$\mathrm{MMP}_{\text {cat }} / \mathrm{EGCG}$ is thus barely attributable to direct inhibition by EGCG. Interestingly, the same experiments reveal that, in comparison with the control without EGCG, the gelatinolytic activity of MT1$\mathrm{MMP}_{\text {cat }}$ in EGCG-treated samples $(69-80 \%$ of control) is much less reduced compared with that of MMP-2 in EGCG-treated samples (22-10\%); it seems very likely that the binding EGCG-MMP-2 is more resistant than EGCG-MT1-MMP cat to the harsh electrophoretic conditions, but this needs further study.

In membrane extracts of HT- 1080 cells cultured 24 hours in the presence of up to $10 \mu \mathrm{M}$ EGCG, the increased levels of both MT1-MMP and the zymogen form of MMP-2, together with an unmodified amount of the MMP-2-activated form (Fig. 5), although not a direct proof, reinforce the hypothesis that the flavanol may indeed exert its direct inhibition on MT1-MMP at the membrane level of living cells. Without impairing 

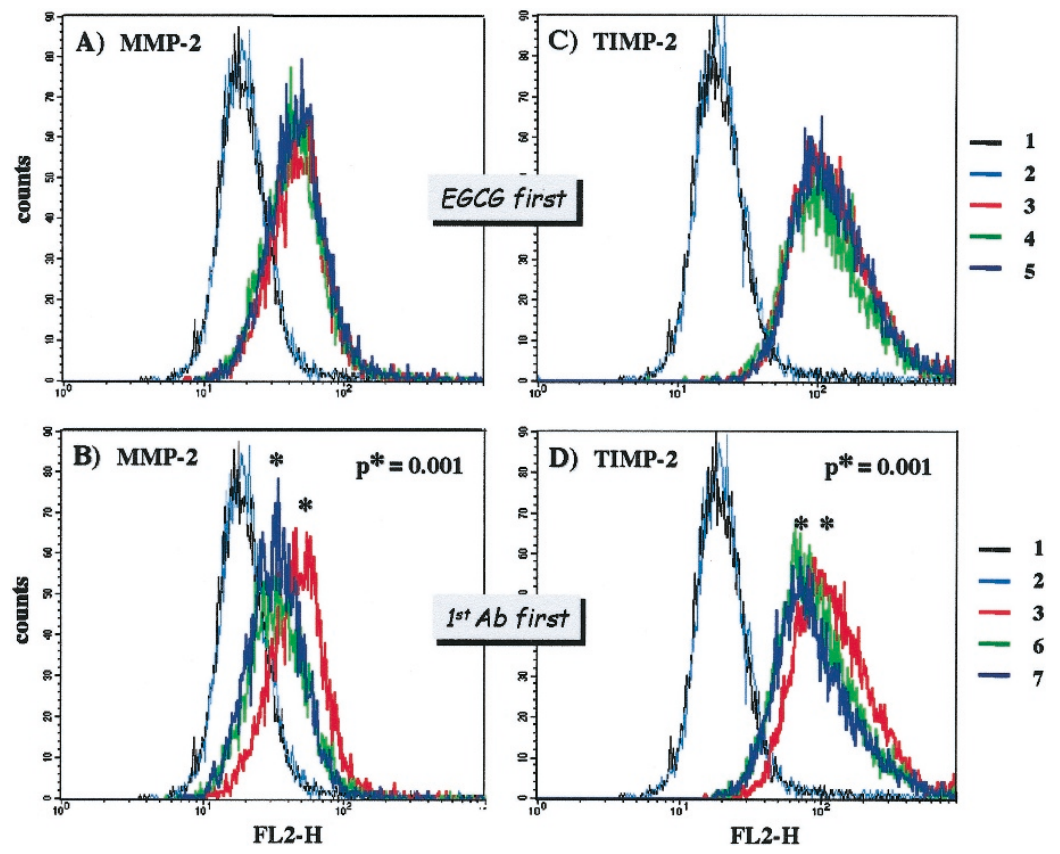

Figure 6.

Cytofluorimetric analyses of MMP-2 and TIMP-2 on HT-1080 cells in the presence or absence of EGCG. (1) without first antibody (Ab) and without EGCG (control), (2) without first $\mathrm{Ab}$ and with $10 \mu \mathrm{M} \mathrm{EGCG} \mathrm{(control),} \mathrm{(3)} \mathrm{with} \mathrm{first} \mathrm{Ab} \mathrm{and} \mathrm{without} \mathrm{EGCG,} \mathrm{(4)} \mathrm{with} \mathrm{first} \mathrm{Ab} \mathrm{given} \mathrm{after} \mathrm{a} \mathrm{1-hour} \mathrm{incubation} \mathrm{with} 1 \mu \mathrm{m} \mathrm{EGCG,} \mathrm{(5)} \mathrm{with}$ first Ab given after a 1-hour incubation with $10 \mu \mathrm{M} E G C G$, (6) with first Ab given before a 1-hour incubation with $1 \mu \mathrm{m} \mathrm{EGCG.} \mathrm{(7)} \mathrm{with} \mathrm{first} \mathrm{Ab} \mathrm{given} \mathrm{before} \mathrm{a} \mathrm{1-hour}$ incubation with $10 \mu \mathrm{M}$ EGCG. The first Ab was anti-MMP-2 in A and B and anti-TIMP-2 in C and D. A moderate but significant decrease in both MMP-2 and TIMP-2 positivity is registered (peaks 6 and 7 versus peak $3, p=0.001$ ) when EGCG treatment followed incubation with first Ab. Example of triplicate experiment.
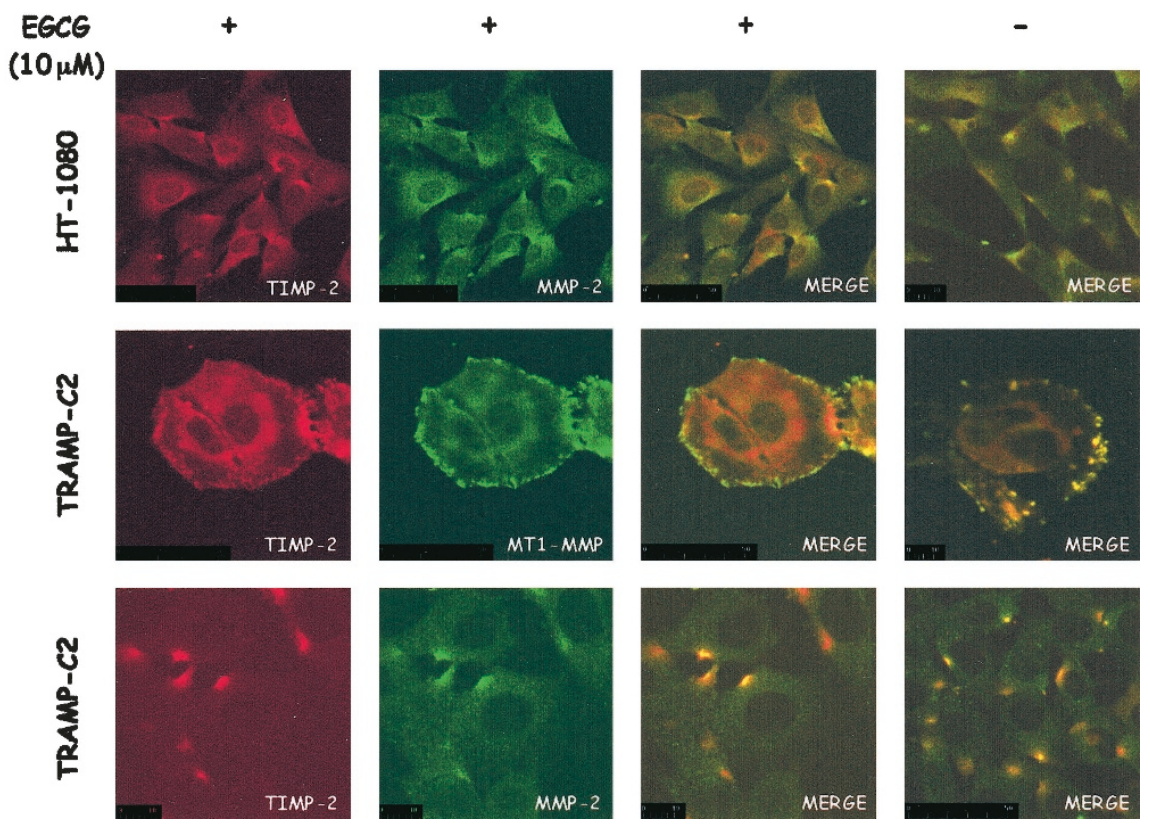

Figure 7.

Colocalization of MT1-MMP, TIMP-2, and MMP-2 in two transformed invasive cell lines treated with (+) and without (-) $10 \mu \mathrm{M}$ EGCG. No detectable effect of EGCG is registered on the localization of the three antigens compared with the control. The shortest scale bar is $10 \mu \mathrm{m}$, the medium and longer ones are $50 \mu \mathrm{m}$.

the potential of binding pro-MMP-2 to its membrane receptor-activator, EGCG may thus restrain, both directly (inhibition of active MMP-2) and indirectly (inhibition of MMP-2 activation), the invasive potential and migration of cells, as described in the glioblastoma cell model (Annabi et al, 2002).
Conversely, at $20 \mu \mathrm{M}$ flavanol, the level of membrane-bound MT1-MMP was comparable with that registered in untreated cells. This may be attributable to the cytotoxicity already reported for phytofactor concentrations $>10 \mu \mathrm{M}$, which causes the culture implosion in our fibrosarcoma model system 
(Garbisa et al, 2001). This may also explain the downregulation of MT1-MMP transcript levels in a different cell type (glioblastoma) reported by others at $25 \mu \mathrm{M}$ (Annabi et al, 2002). In both cases, restraint in proMMP-2 activation was equally registered (cellular, here; secreted, Annabi et al, 2002).

But other cellular events may result from inhibition of MT1-MMP. Recent studies demonstrate that MT1MMP autocatalytic shedding may represent a mechanism to terminate MT1-MMP activity, while the nonautocatalytic shedding generates soluble active MT1MMP capable of binding TIMP-2. This suggests that the pericellular and extracellular activities of MT1MMP are regulated through a delicate balance of active and inactive enzyme soluble fragments (Toth et al, 2002) and binding to TIMP-2.

We thus extended the study through an immunocytological investigation of whether the exogenous inhibitor EGCG affects TIMP-2/MMP-2 binding to MT1MMP in intact cells. We demonstrated that the interaction of the flavanol with MT1-MMP does not affect the persistence of the MT1-MMP/TIMP-2/ MMP-2 trimer on the cell membrane. This was deduced from cytofluorimetric analyses of cells treated with EGCG - up to $10 \mu \mathrm{M}$-before and after contact with specific antibodies. In the first case, the cell positivity for TIMP-2 and MMP-2 remained unmodified, compared with the control counterpart (Fig. 6, A and $\mathrm{C}$ ); in the second, the positivity-mainly that of MMP-2-was slightly but significantly $(p=0.001)$ down-modulated (Fig. 6, B and D), indicating a partial interference of EGCG with the (monoclonal) antibody/ antigen binding. This latter point may be somehow indicative of the MMP-2 and TIMP-2 molecular region-or one of them-where EGCG binds, in this case, the 468 to 483 and 178 to 193 epitopes, respectively. This approach, with the use of a series of monoclonal antibodies for different epitopes, may add information about the EGCG/target molecule interaction, useful for both understanding the EGCG reactivity and designing potentially more powerful molecules (Sartor et al, 2002a).

The confocal immunolocalization of the antigens on human (HT-1080) and murine (TRAMP-C2) invasive tumor cells confirms that, in the presence of EGCG, the components of the trimer remain colocalized, mostly concentrated in just a few protruding cellular cones or peripheral caveolae, as already reported (Puyraimond et al, 2001). Thus, the phytofactor does not disassemble the MT1-MMP/TIMP-2/MMP-2 aggregate nor modify its membrane microdomains, where matrix proteolysis is restricted by default to a limited microenvironment at the cell surface. Recent evidence suggests that, under certain conditions, the presence of synthetic inhibitors stabilizes MT1-MMP on the cell surface, while the absence or reduced levels of inhibitors facilitate autocatalysis and shedding of MT1-MMP (Bernardo et al, 2002; Toth et al, 2000).

This phytofactor has thus the potential to "hinder the molecular shears" instrumental to tumor invasion (Garbisa et al, 1999) through multiple actions: It di- rectly inhibits the proteolytic activity of MT1-MMP, potentially restraining the activation of matrix protease MMP-2, MMP-9, and MMP-13; it directly inhibits the proteolytic activity of MMP-2 and MMP-9 on matrix components (Demeule et al, 2000; Garbisa et al, 1999); and it leaves engaged the receptor (MT1MMP) with a "double blunted" MMP-2 (nonactivated and/or inhibited).

This scenario may explain in part the lower $\mathrm{IC}_{50}(0.1$ $\mu \mathrm{M})$ registered for EGCG in in vitro cell invasion assays, where all the mentioned events can take place, in comparison with the $\mathrm{IC}_{50}$ individually measured for MMP-2, MMP-9, and MT1-MMP by zymography $(10,30$, and $5 \mu \mathrm{M}$, respectively) (Garbisa, 2001).

Considering the growing number of new functions for the matrix metallo-proteinases in cancer progression (Egeblad and Werb, 2002), the effect of the catechin on the membrane-controlled proteases may represent also a key molecular event up-stream of some other cellular outcomes following EGCG treatment, such as cell cycle arrest (Ahmad et al, 2000) and apoptosis (Chung et al, 2001). The interaction with the catechin may indeed affect the cytoplasmic C-terminus of MT1-MMP, which in turn could play a crucial role in some signal transduction pathways. The possibility of modulating this and other cascade events controlled by MT1-MMP by an orally available natural product is thus very promising for treating proliferative and invasive pathologies and deserves further investigation.

\section{Materials and Methods}

\section{Cells}

HT-1080 human fibrosarcoma cells (Mazzieri, 1997) were routinely grown in Dulbecco's modified Eagle's medium (DMEM) (Flow Laboratories, United Kingdom) supplemented with $10 \%$ heat-inactivated fetal calf serum (hi-FCS) (Biochrom, Berlin, Germany); TRAMP-C2 mouse prostate carcinoma cells were grown in dihydrotestosterone- and other supplement-containing DMEM, as described (Ghosh et al, 1999). In some experiments, gelatinase-free hi-FCS (see below) was used instead of hi-FCS. Both media were supplemented with $100 \mathrm{U} / \mathrm{ml}$ penicillin and $100 \mu \mathrm{g} / \mathrm{ml}$ streptomycin (Labtek Eurobio, Corsio, Italy), and the cells were incubated in $5 \% \mathrm{CO}_{2}$ in air at $37^{\circ} \mathrm{C}$. All experiments were initiated with cells in the log phase of growth with at least $25 \%$ confluence and were designed to be completed before $80 \%$ confluence. A $1 \mathrm{~mm}$ stock solution of (-)epigallocatechin-3gallate (EGCG) was freshly prepared in culture medium and added to cultures at the reported concentration. At the end of the incubation (24 hours), the cells were harvested and the membrane proteins extracted as described below.

\section{Antibodies}

The following antibodies were used for Western blotting, immunofluorescence, and cytofluorimetry-primary antibodies (all containing $0.1 \%$ sodium azide): (1) mouse anti-MMP-2 (epitope: amino acids 468-483 of 
human, numbered from pro-peptide) from Oncogene, (2) mouse anti-MT1-MMP (epitope: amino acids 319333 in the hemopexin-like domain of human, numbered from the signal peptide) from Oncogene, (3) mouse anti-MT1-MMP (oligo-peptide 160-173 on human) from Chemicon, (4) mouse anti-TIMP-2 (epitope: amino acids $178-193$ of human) from Oncogene, and (5) chicken anti-human TIMP-2 (complete); secondary antibodies: goat anti-mouse IgG peroxidase conjugated (Chemicon) for Western blotting, goat antimouse IgG fraction FITC conjugated (Sigma, St. Louis, Missouri), sheep anti-rabbit IgG Cy3 conjugated (Sigma), rabbit anti-chicken IgG TRITC conjugated (Sigma) for immunolocalization; goat anti-mouse $\operatorname{lgG}-1(\gamma)$ R-PE conjugated (Caltag Laboratories, Burlingame, California) for cytofluorimetry. As the isotype control, mouse IgG1 (Becton Dickinson, San José, California) were used at $1 \mu \mathrm{l} / 5 \times 10^{5}$ cells.

\section{FCS Treatment}

EGCG-containing culture medium was supplemented with gelatinase-free hi-FCS. The latter was used to detect gelatinases exclusively contributed by the cells in the presence of serum and was prepared by adding $20 \% \mathrm{v} / \mathrm{v}$ of gelatin-sepharose (Amersham Pharmacia, Biotech, United Kingdom) to hi-FCS. After gentle mixing at $4^{\circ} \mathrm{C}$ for 2 hours and centrifugation at $100 \times g$ for 5 minutes, the supernatant and pellet were analyzed by gelatin zymography to verify removal of all gelatinases from the serum.

\section{MT1-MMP Activity on Synthetic Substrate}

Soluble complete pro-MT1-MMP from Escherichia coli was purchased from Oncogene and assayed as described (Knight et al, 1992) using the fluorogenic substrate Mca-Pro-Leu-Gly-Leu-Dpa-Ala-Arg- $\mathrm{NH}_{2}$ (BACHEM AG, Switzerland). The pro-MT1-MMP was activated following the method described by StetlerStevenson et al (1989): Aliquots of zymogen were incubated at $4^{\circ} \mathrm{C}$ with $1 \mathrm{~mm}$ final concentration of p-APMA (Sigma) for 15 hours. The substrate was added, the mixture incubated at $37^{\circ} \mathrm{C}$, and fluorimetric analysis carried out in Perkin-Elmer MPF4 spectrophotofluorimeter $\left(\lambda_{\text {ex }} 328 \mathrm{~nm}, \lambda_{\text {em }} 393 \mathrm{~nm}\right)$. The emission of appropriate controls was subtracted.

\section{Cell Membrane Extraction}

After rinsing with PBS, the cells were harvested with 2 mM EDTA, centrifuged 5 minutes at $500 \times g$, and rinsed twice with PBS. The pellet was resuspended in $200 \mu \mathrm{l}$ of $0.1 \%$ digitonin (Sigma), $0.5 \mathrm{M} \mathrm{NaCl}, 20 \mathrm{~mm}$ Hepes, pH 7 (DIG buffer 1), and incubated 10 minutes at $4^{\circ} \mathrm{C}$. Following a 5-minute centrifugation at 1200 $\times g$, the pellet was rinsed three times with DIG buffer 1 , resuspended in $1 \%$ digitonin, $0.15 \mathrm{M} \mathrm{NaCl}, 20 \mathrm{~mm}$ Hepes, pH 7 (DIG buffer 2), and incubated 2 hours at $4^{\circ} \mathrm{C}$ with gentle motion. The sample was then centrifuged 5 minutes at $1200 \times g$ to collect the cell membrane protein-containing supernatant.

\section{Zymographic Analysis}

EGCG effects on MT1-MMP gelatinolytic activity were studied using the human recombinant catalytic domain of MT1-MMP (MT1-MMP ${ }_{\text {cat }}$ ), comprising the Tyr $^{89}$-Gly ${ }^{265}$ residues (MT1-MMP ${ }_{\text {cat }}$ ), from Escherichia coli, purchased from Calbiochem. Aliquots of MT1MMP $(40 \mu \mathrm{U})$ were assayed by gelatin zymography developed in the presence of increasing amounts of EGCG (0.01-1000 $\mu \mathrm{M})$. Without heating, samples underwent electrophoresis in $0.1 \%$ gelatin containing $13 \%$ polyacrylamide gels in the presence of SDS under nonreducing conditions, as described in Garbisa et al (2001). After electrophoresis, the gels were washed twice for 15 minutes with $2.5 \%$ Triton X-100 and incubated overnight at $37^{\circ} \mathrm{C}$ in $50 \mathrm{~mm}$ Tris- $\mathrm{HCl}$, $200 \mathrm{~mm} \mathrm{NaCl}, 10 \mathrm{~mm} \mathrm{CaCl}_{2}$, pH 7.4 (buffer 1) containing EGCG. The gels were then stained 30 minutes with $30 \%$ methanol $/ 10 \%$ acetic acid containing $0.5 \%$ Coomassie Brilliant Blue R-250 and destained in the same solution without dye; clear bands represent areas of gelatinolysis on the blue background. Digestion bands were quantitated by an image analyzer system with GelDoc 2000 and Quantity One software (BioRad, Hercules, California).

To study the activity of MT1-MMP $\mathrm{Mat}_{\text {cat }}$ on proMMP-2, pro-MMP-2-containing culture medium, conditioned by SK-N-BE human neuroblastoma cells (Negro et al, 1997), was preincubated 30 minutes at $4^{\circ} \mathrm{C}$ with $1 \mathrm{~mm}$ PMSF and $5 \mathrm{~mm} \mathrm{NEM} ; 8$ to $40 \mu \mathrm{U}$ MT1-MMP ${ }_{\text {cat }}$ in buffer 1 were added, and the mixture was incubated for 2 and 4 hours and analyzed for gelatinolytic activities using $8 \%$ polyacrylamide gels.

To study the effect of EGCG on pro-MMP-2 activa-

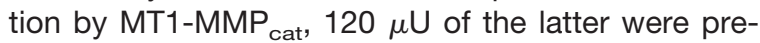
mixed with EGCG $(20$ and $60 \mu \mathrm{M})$ for 20 minutes at $4^{\circ} \mathrm{C}$ in buffer 1 ; the mixture was then added to pro-MMP-2-containing medium pretreated with PMSF and NEM (as above). Samples of medium alone, without EGCG, and without MT1-MMP cat were used as controls. The samples were incubated 4 hours at $33^{\circ} \mathrm{C}$ and directly examined by $8 \%$ polyacrylamide gelatin zymography.

\section{Western Blotting}

The protein content of lysed cell samples was directly evaluated by Bradford (Pierce) and the volume adjusted to equal concentration. As control proteins, human recombinant MT1-MMP and pro-MMP-2containing culture medium (as above) were used. Following SDS-PAGE under reducing conditions, samples were electroblotted to a Hybond-C Extra nitrocellulose membrane (Amersham). The membrane was prehybridized at room temperature (RT) for 2 hours in PBS with $0.1 \%$ Tween-20 (PBS-T) containing $1 \%$ nonfat dried milk (Sigma) (sol.1) and hybridized at RT for 1 hour in PBS-T containing 3\% BSA and the first antibody [anti-MT1-MMP (antibody 3) and antiMMP-2 (antibody 1), as detailed in the "Antibodies" paragraph above]. The membrane was then incubated at RT for 45 minutes in sol. 1 containing the second 
antibody 1:2000 (as detailed in the "Antibodies" paragraph above). Each step was followed by extensive washing in PBS-T. Antigen detection was achieved by incubating the membrane for 1 minute at RT with $0.125 \mathrm{ml} / \mathrm{cm}^{2}$ of ECL detection solution and exposing to Hyperfilm MP (both from Amersham Pharmacia) for 10 seconds up to 5 minutes.

\section{Confocal Immunofluorescence Microscopy}

Cells were seeded on glass coverslips and, after 17 hours of incubation at $37^{\circ} \mathrm{C}$ in $5 \% \mathrm{CO}_{2}$, fixed with $4 \%$ paraformaldehyde for 30 minutes. The cells were subsequently treated as follows: After washing with PBS and blocking with $0.3 \%$ BSA in PBS, they were incubated with a mix of two first antibodies (anti-MT1MMP, antibody 2 in the "Antibody" paragraph above; anti-TIMP-2, antibody 5; anti-MMP-2, antibody 1) overnight at $4^{\circ} \mathrm{C}$, washed four times with PBS, incubated with the appropriate secondary antibodies for 1 hour at RT, and washed four times with PBS. Coverslips were then mounted on microscope slides over a drop of fluorescent mounting medium (Dako). Staining was visualized using a Bio-Rad confocal microscope.

\section{Cytofluorimetry}

Cells in log phase of growth were harvested using only EDTA (2 $\mathrm{mm}$ in PBS without $\mathrm{Ca}^{2+}$ and $\mathrm{Mg}^{2+}$ ) for 3 minutes at $37^{\circ} \mathrm{C}$, pelleted, and rinsed twice in PBS with $\mathrm{Ca}^{2+}$ and $\mathrm{Mg}^{2+}$. The cells were resuspended $\left(10^{6} / 200\right.$ $\mu l)$ in PBS and incubated 1 hour at RT with 1 and $10 \mu \mathrm{M}$ EGCG; after rinsing with PBS, they were incubated with mouse first antibody (anti-MMP-2, antibody 1; antiTIMP-2, antibody 4) under gentle motion. Alternatively, the incubation with the first antibody preceded treatment with EGCG, following the same time schedule. The cells were then rinsed, the second antibody added (antimouse IgG1 PE), and the suspension incubated as above for 30 minutes. After rinsing, the frequency of positive cells was determined by overlaying the flow cytometry histograms of the samples. Cells were scored using a FACalibur analyzer (Becton-Dickinson), and the data were processed using the Macintosh CELLQuest software program (Becton-Dickinson). To evaluate whether the shift of the positive cell peak was statistically significant, the Kolmogorov-Smirnov test for histogram analysis was used following the user's guide to the above program.

\section{Acknowledgements}

We are grateful to Prof. F. Ricchelli and Prof. G. Jori (Department of Biology, University of Padova) and Dr. A. Negro (Department of Biological Chemistry, University of Padova) for helpful contributions, Mr. D. Carollo (Department of Experimental Clinical Immunology, Medical School of Padova) for assistance in cytofluorimetry, Dr. W. G. Stetler-Stevenson for his generous gift of chicken anti-human TIMP-2 antibodies, Dr. E. Kwon for his generous gift of TRAMP-C2 cells, and Dr. $S$ Biggin for revision of the English manuscript.

\section{References}

Ahmad N, Cheng P, and Mukhtar H (2000). Cell cycle disregulation by green tea polyphenol epigallocatechin-3gallate. Biochem Biophys Res Commun 275:328-334.

Annabi B, Lachambre M-P, Bousquet-Gagnon N, Pagè M, Gingras D, and Béliveau R (2002). Green tea polyphenol (-)-epigallocatechin 3-gallate inhibits MMP-2 secretion and MT1-MMP-driven migration in glioblastoma cells. Biochim Biophys Acta 1542:209-220.

Bernardo MM, Brown S, Li ZH, Fridman R, and Mobashery S (2002). Design, synthesis, and characterization of potent, slow-binding inhibitors that are selective for gelatinases. J Biol Chem 277:11201-11207.

Caenazzo C, Onisto M, Sartor L, Scalerta R, Giraldo A, Nitti $D$, and Garbisa S (1998). Augmented membrane type 1 matrix metallo-proteinase (MT1-MMP): MMP-2 messenger RNA ratio in gastric carcinomas with poor prognosis. Clin Cancer Res 4:2179-2186.

Cao $Y$ and Cao R (1999). Angiogenesis inhibited by drinking tea. Nature 398:381.

Chung LY, Cheung TC, Kong SK, Fung KP, ChoyYM, Chan ZY, and Kwok (2001). Induction of apoptosis by green tea catechins in human prostate cancer DU145 cells. Life Sci 68:1207-1214.

Cowell S, Knauper V, Stewart ML, D'Ortho MP, Stanton H, Hambry RM, Lopez-Otin C, Reynolds JJ, and Murphy G (1998). Induction of matrix metalloproteinase activation cascade based on membrane-type 1 matrix metalloproteinase: Associated activation of gelatinase A, gelatinase $B$ and collagenase 3. Biochem J 331:453-458.

Demeule M, Brossard M, Page M, Gingras D, and Béliveau R (2000). Matrix metalloproteinase inhibition by green tea catechins. Biochim Biophys Acta 1478:51-60.

Egeblad M and Werb Z (2002). New functions for the matrix metalloproteinases in cancer progression. Nat Rev 2:161174.

Garbisa S, Biggin S, Cavallarin N, Sartor L, Benelli R, and Albini A (1999). Tumor invasion: Molecular shears blunted by green tea. Nature Med 4:1216.

Garbisa S, Sartor L, Biggin S, Salvato B, Benelli R, and Albini $A$ (2001). Tumor gelatinases and invasion inhibited by green tea flavanol epigallocatechin-3-gallate. Cancer 91:822-832.

Ghosh PM, Ghosh-Choudhury N, Moyer ML, Mott GE, Thomas CA, Foster BA, Greenberg NM, and Kreisberg JI (1999). Role of RhoA activation in the growth and morphology of a murine prostate tumor cell line. Oncogene 18:41204130 .

Knight GC, Willenbrock F, and Murphy G (1992). A novel cumarin-labelled peptide for sensitive continuous assays of the matrix metalloproteinases. FEBS Lett 296:263-266.

Liotta LA, Tryggvason K, Garbisa S, Hart I, Foltz CM, and Shafie S (1980). Metastatic potential correlates with enzymatic degradation of basement membrane collagen. Nature 284:67-68.

Maeda-Yamamoto M, Kawahara H, Tahara N, Tsuji K, Hara $\mathrm{Y}$, and Isemura M (1999). Effects of tea polyphenols on the invasion and matrix proteinases activities of human fibrosarcoma HT-1080 cells. J Agric Food Chem 47:2350-2354. 
Mazzieri R, Masiero L, Zanetta L, Monea S, Onisto M, Garbisa S, and Mignatti P (1997). Control of type IV collagenase activity by components of the urokinase-plasmin system: A regulatory mechanism with cell-bound reactants. EMBO J 16:101-104.

Negro A, Onisto M, Pellati D, and Garbisa S (1997). CNTF up-regulation of TIMP-2 in neuroblastoma cells. Mol Brain Res 48:30-36.

Pei D and Weiss SJ (1996). Transmembrane-deletion mutants of the membrane-type matrix metalloproteinase- 1 process progelatinase $A$ and express intrinsic matrix-degrading activity. J Biol Chem 271:9135-9140.

Puyraimond A, Fridman R, Lemesle M, Arbeille B, and Menashi S (2001). MMP-2 colocalizes with caveolae on the surface of endothelial cells. Exp Cell Res 262:28-36.

Sartor L, Pezzato E, Dell'Aica I, Caniato R, Biggin S, and Garbisa S (2002a). Inhibition of matrix proteases by polyphenols: Chemical insights for anti-inflammatory and anti-invasion drug design. Biochem Pharmacol 64:229-237.

Sartor L, Pezzato E, and Garbisa S (2002b). (-)Epigallocatechin-3-gallate inhibits leukocyte elastase: Phytofactor for hindering inflammation, emphysema and invasion. J Leuk Biol 71:73-79.

Sato H, Takino T, Okada Y, Cao J, Shinagawa A, Yamamoto E, and Seiki M (1994). A matrix metalloproteinase expressed on the surface of invasive tumor cells. Nature 370:61-65.

Sazuka M, Murakami S, Isemura M, Satoh K, and Nukiwa T (1995). Inhibitory effects of green tea infusion on in vitro invasion and in vivo metastasis of mouse lung carcinoma cells. Cancer Lett 98:27-31.

Sternlicht MD and Werb Z (1999). ECM proteinases. In: Kreis $\mathrm{T}$ and Vale $\mathrm{R}$, editors. Extracellular matrix, anchor, and adhesion proteins. Oxford: Oxford University Press, 503563.

Stetler-Stevenson W, Krutzsch HC, Wacher MP, Margulies IM, and Liotta LA (1989). The activation of human type IV collagenase proenzyme. Sequence identification of the major conversion product following organomercurial activation. J Biol Chem 264:1353-1356.
Taniguchi S, Fujiki H, Kobayashi H, Go H, Miyado K, Sadano $H$, and Shimokawa R (1992). Effect of (-)-epigallocatechin gallate, the main constituent of green tea, on lung metastasis with mouse B16 melanoma cell lines. Cancer Lett 65:51-54.

Toth M, Bernardo MM, Gervasi DC, Soloway PD, Wang Z, Bigg HF, Overall CM, DeClerck YA, Tschesche H, Cher ML, Brown S, Mobashery S, and Fridman R (2000). Tissue inhibitor of metalloproteinase (TIMP)-2 acts synergistically with synthetic matrix metalloproteinase (MMP) inhibitors but not with TIMP-4 to enhance the (membrane type 1)-MMPdependent activation of pro-MMP-2. J Biol Chem 275: 41415-41423.

Toth M, Hernandez-Barrantes S, Osenkowski P, Bernardo MM, Gervasi DC, Shimura Y, Meroueh O, Kotra LP, Gàlvez BG, Arroyo AG, Mobashery S, and Fridman R (In press, 2002). Complex pattern of membrane type 1-matrix metalloproteinase shedding. Regulation by autocatalytic cell surface inactivation of active enzyme. J Biol Chem.

Wang ZY, Cheng SJ, Zhou ZC, Athar M, Khan WA, Bickers DR, and Mukhtar H (1989). Antimutagenic activity of green tea polyphenols. Mutat Res 223:273-285.

Wang ZY, Hong JY, Huang MT, Reuhl KR, Conney AH, and Yang CS (1992). Inhibition of N-nitrosodiethylamine- and 4-(methylnitrosamino)-1-(3-pyridyl)-1-butanone-induced tumorigenesis in $\mathrm{A} / \mathrm{J}$ mice by green tea and black tea. Cancer Res 52:1943-1947.

Yang CS, Landau JM, Huang MT, and Newmark HL (2001). Inhibition of carcinogenesis by dietary polyphenolic compounds. Annu Rev Nutr 21:381-406.

Yang CS and Wang Z-Y (1993). Tea and cancer. J Natl Cancer 85:1038-1049. 